\title{
Influence of Cow's Milk and Esomeprazole on the Absorption of Erlotinib: A Randomized, Crossover Pharmacokinetic Study in Lung Cancer Patients
}

\author{
G. D. Marijn Veerman ${ }^{1} \cdot$ Koen G. A. M. Hussaarts ${ }^{1} \cdot$ Robert Peric $^{2} \cdot$ Esther Oomen-de Hoop $^{1} \cdot$ Kersten D. Landa $^{1}$. \\ Cor H. van der Leest ${ }^{3}$. Suzanna D. Broerse $e^{4}$ Hugo B. Rutten ${ }^{5}$. Huub N. A. Belderbos ${ }^{3}$. Christi M. J. Steendam ${ }^{2,3}$. \\ Marthe S. Paats ${ }^{2}$. Stijn L. W. Koolen ${ }^{1,6} \cdot$ Anne-Marie C. Dingemans ${ }^{2} \cdot$ Teun van Gelder ${ }^{6} \cdot$ Roelof W. F. van Leeuwen $^{1,6}$. \\ Joachim G. J. V. Aerts ${ }^{2}$ Ron H. J. Mathijssen ${ }^{1}$
}

Published online: 17 June 2020

(c) The Author(s) 2020

\begin{abstract} (40 $\mathrm{mg}$ once daily $3 \mathrm{~h}$ prior to erlotinib) was taken for 3 days. were observed between milk and water. $\mathrm{AUC}_{24}$ and $C_{\max }$ and should be avoided if possible.

\section{Introduction}

Erlotinib is a tyrosine kinase inhibitor (TKI) registered for the treatment of epidermal growth factor receptor (EGFR)mutated metastatic non-small cell lung cancer (NSCLC) [1, 2]. It is indicated in combination with gemcitabine as firstline therapy for unresectable or metastatic pancreatic cancer [1]. Erlotinib is orally administered on a daily basis at a dose of 150 and $100 \mathrm{mg}$ once daily for NSCLC and pancreatic
\end{abstract}

Introduction Erlotinib's gastrointestinal solubility and absorption are decreased by proton pump inhibitors (PPIs). Since erlotinib is a lipophilic drug, we hypothesized that concomitant intake with the fatty beverage milk may be a feasible way to increase erlotinib uptake. We performed a two-period, randomized, crossover study to investigate the influence of cow's milk with 3.9\% fat on the exposure of erlotinib with and without the PPI esomeprazole in patients with non-small cell lung cancer (NSCLC). The effect of esomeprazole was studied in an additional intrapatient comparison.

Method Pharmacokinetic sampling was performed on days 7 and 14 during 24 consecutive hours. During the 7 days prior to pharmacokinetic sampling, erlotinib was taken daily with $250 \mathrm{~mL}$ of either water or milk. In the PPI arm, esomeprazole

Results Erlotinib area under the curve from time zero to $24 \mathrm{~h}\left(\mathrm{AUC}_{24}\right)$ did not significantly change when administered with milk, compared with water, in both non-PPI users $(n=14 ;-3 \%$; 95\% confidence interval $[\mathrm{CI}]-12$ to $8 \% ; p=0.57)$ and patients who used esomeprazole $(n=15 ; 0 \% ; 95 \% \mathrm{CI}-15$ to $17 \% ; p=0.95)$. Esomeprazole decreased erlotinib $\mathrm{AUC}_{24}$ by $47 \%(n=9 ; 95 \% \mathrm{CI}-57$ to $-34 \% ; p<0.001)$ and $C_{\max }$ by $56 \%(95 \% \mathrm{CI}-64$ to $-46 \% ; p<0.001)$. No differences in toxicities

Conclusion Milk with 3.9\% fat has no effect on the exposure to erlotinib in NSCLC patients, independent of PPI use. The combination with milk is safe and well tolerated. Concomitant esomeprazole treatment strongly decreased both erlotinib

G. D. Marijn Veerman and Koen G. A. M. Hussaarts contributed equally to this work.

G. D. Marijn Veerman

g.veerman@erasmusmc.nl

Extended author information available on the last page of the article cancer, respectively. Intra- and interpatient variability differs significantly due to interactions with food [3], concomitant medication [4], and lifestyle factors (i.e. smoking) [5, 6].

The bioavailability of erlotinib largely depends on its solubility in the stomach and passive diffusion and probable active cellular transport in the gastrointestinal tract [7]. Optimal drug absorption is reached at a physiologically low intragastric $\mathrm{pH}$ (i.e. $\mathrm{pH}$ value of 1 ), since erlotinib is then protonized and is thus better soluble [8]. However, various acid-reducing drugs, including histamine- 2 receptor antagonists (e.g. ranitidine) and proton pump inhibitors (PPIs; e.g. omeprazole) may lead to a $40-50 \%$ decrease in erlotinib absorption due to an increase in intragastric $\mathrm{pH}$ [9]. It has been previously demonstrated that this impaired systemic exposure to erlotinib can be corrected when administered in combination with the acidic beverage cola [10]. However, daily intake of acidic and highly caloric beverages such as 


\section{Key Points}

Cow's milk did not significantly alter the exposure of erlotinib, while esomeprazole decreased both the area under the curve from time zero to $24 \mathrm{~h}$ and maximum concentration of erlotinib by $47 \%$ and $56 \%$, respectively.

Patient-reported toxicity was equal between the milk and water groups, with and without proton pump inhibitors.

Hence, cow's milk can be used as a safe alternative to water for the administration of erlotinib. Concomitant treatment with esomeprazole should be avoided if possible.

cola or orange juice has disadvantages, such as dental problems, disrupted bone mineral composition, and weight gain [11]. We hypothesized that a healthier way to enhance erlotinib bioavailability could be by making use of the effects of other food components. The exposure of erlotinib is increased 33-66\% when administered concomitantly with a high-fat meal [3]. We explored this potentially positive food effect as a proof-of-principle by optimizing erlotinib absorption in the presence of a beverage containing fat. In the past, milk-based drug formulations have shown to be equally effective compared with standard formulations in terms of solubility and dispersion [12]. Milk is consumed worldwide by billions of people. It is a healthy beverage that contains essential proteins, vitamins and minerals (e.g. calcium and phosphorus). Cow's milk accounts for more than $80 \%$ of the global milk production [13].

This is the first study that investigates the effects of erlotinib administered concomitantly with high-fat whole cow's milk compared with water. In addition, a direct intrapatient comparison to study the effects of esomeprazole on the systemic exposure of erlotinib has never been made. Therefore, we also explored the potential drug-drug interaction of esomeprazole use on the absorption of erlotinib.

\section{Methods}

\subsection{Patient Eligibility}

Adult NSCLC patients were eligible for inclusion in this study if they had an Eastern Cooperative Oncology Group (ECOG) performance status [14] of $\leq 1$, were treated with a stable dose of erlotinib for at least 2 weeks (to guarantee steady-state plasma concentrations) and did not use any other (complementary or alternative) medicine or compounds that may have the potential to interact with either erlotinib or esomeprazole. Patients who concomitantly used any prescribed PPI could only participate in the PPI arm of this study when willing to switch to esomeprazole. It was possible for patients to participate in both study arms if PPI use was discontinued or if they were willing to take esomeprazole as required for this study. All participating patients were asked to sign a written informed consent form. The study was approved by the local Ethics Committee (Erasmus University Medical Center Rotterdam; MEC 16-590) and was registered in the Dutch Trial Registry (number NL5984; NTR6148) [15].

\subsection{Study Design}

This was a single center, randomized, two-period, crossover pharmacokinetic study with two study arms. Figure 1 shows the study flow chart. After signing informed consent and after screening, patients were allocated to the nonPPI (arm A) or PPI (arm B) study arms. Hereafter, they were randomized to start with erlotinib with $250 \mathrm{~mL}$ of water (period 1) or cow's milk containing 3.9\% fat (period 2 ) for 7 consecutive days (days $1-7$ or $8-14$ ). The 7 -day period was chosen to ensure that erlotinib concentrations reached steady state. At days 7 and 14, patients were electively admitted for 24-h pharmacokinetic blood sampling. During each admission, 13 blood samples were collected; $<5 \mathrm{~min}$ before erlotinib intake $(t=0 \mathrm{~h})$ and at time points $(0.5,1,1.5,2,2.5,3,3.5,4,6,8,12$ and $24 \mathrm{~h})$ after erlotinib intake. Patients had to take erlotinib according to its label, i.e. fasted for at least $2 \mathrm{~h}$ prior to and $1 \mathrm{~h}$ after administration. Additionally, on the day of hospital admission, food intake was prohibited between $4 \mathrm{~h}$ prior to and $1 \mathrm{~h}$ after erlotinib administration. Consumption of beverages was restricted for $1 \mathrm{~h}$ before and after erlotinib intake. In the PPI arm, patients were required to take esomeprazole (40 mg once daily) $3 \mathrm{~h}$ prior to erlotinib intake on days 5, 6 and 7 and days 12, 13 and 14 after the start of the study. The timing of esomeprazole intake was chosen to ensure maximal inhibition of gastric acid secretion at the time of erlotinib intake [16]. All samples were analyzed by a validated liquid chromatography-tandem mass spectrometric assay for precise quantification of erlotinib plasma concentrations [17].

\subsection{Study Objectives}

The primary objective was the difference in geometric mean of the area under the curve from time zero to $24 \mathrm{~h}$ (AUC ${ }_{24}$ ) between periods with concomitant cow's milk compared with water, both with and without esomeprazole. Secondary objectives were the effects of esomeprazole intake in patients who were included in both arms, other pharmacokinetic 


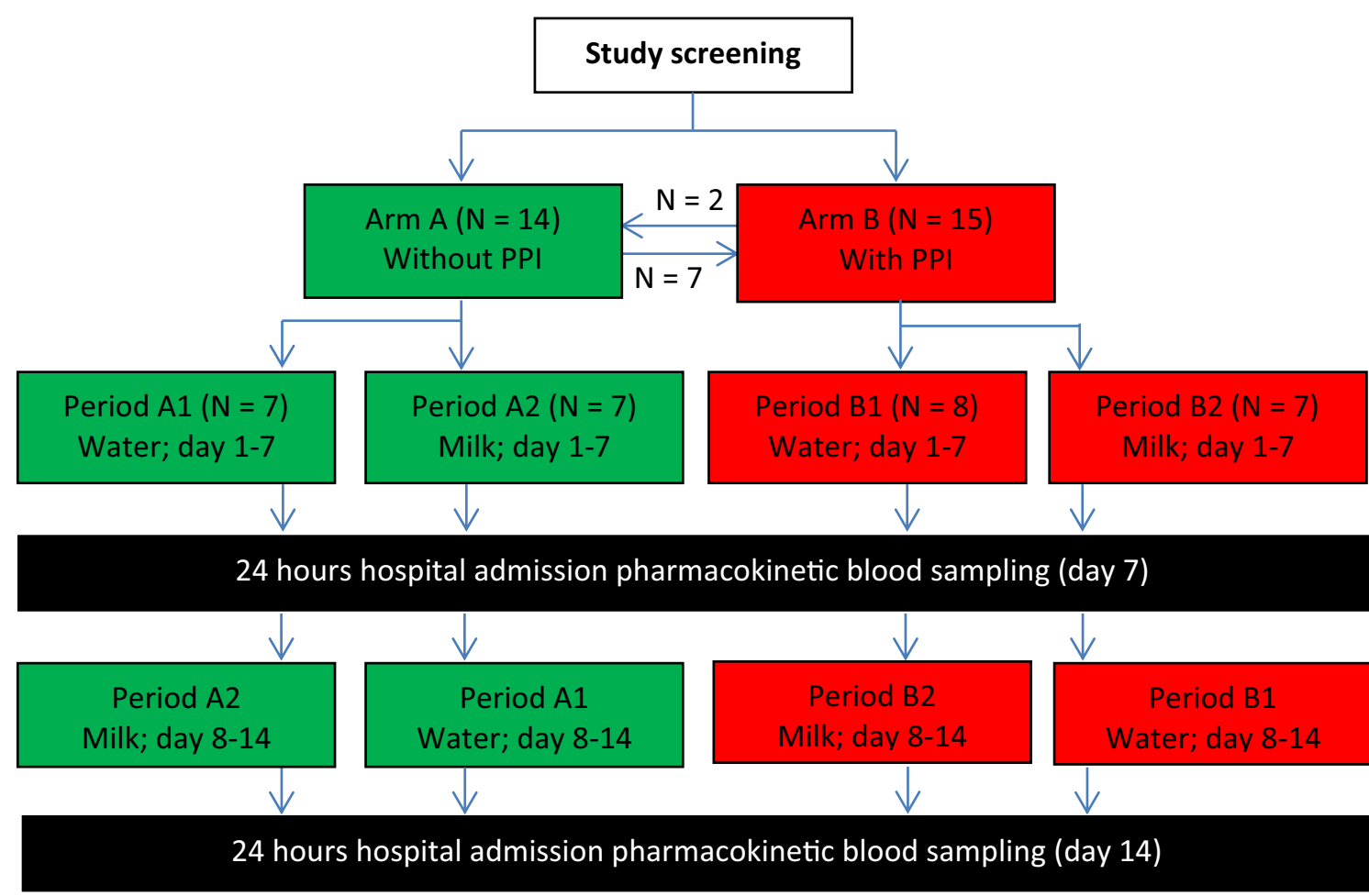

Fig. 1 Study flowchart. After screening, patients were allocated to the non-PPI ( $\operatorname{arm}$ A) or PPI (arm B) arms. Hereafter, they were randomized to start with administration of either concomitant water (period 1) or cow's milk (period 2). Subsequent participation in both arms was allowed and is illustrated with the arrows between arms A

outcomes (i.e. clearance, maximum concentration $\left[C_{\max }\right]$ and time to $C_{\max }\left[T_{\max }\right]$ ), and comparison of (the incidence and severity of) the adverse effects of treatment with erlotinib between periods and study arms.

\subsection{Adverse Event Monitoring}

Toxicity was scored by the investigator at baseline and during hospital admission in accordance with the US National Cancer Institute Common Terminology Criteria for Adverse Events (CTCAE) grades, version 4.03 [18]. Patients were provided with a diary to report any (ongoing) adverse events during the study.

\subsection{Statistical Analyses}

Given a clinically relevant difference of $30 \%$ in AUC, a within-patient standard deviation of $25 \%, 80 \%$ power and a two-sided significance level of 5\%, 14 evaluable patients were required per study group (i.e. with or without esomeprazole) [19]; hence, a total of 28 patients had to be included. and B. Hospital admissions for pharmacokinetic blood sampling took place at days 7 and 14. Esomeprazole $40 \mathrm{mg}$ once daily was administered in arm B at days 5, 6 and 7, and days 12, 13 and 14. PPI proton pump inhibitor

Analyses of $\mathrm{AUC}_{24}$ and $C_{\max }$ were performed on logtransformed values, since these parameters were assumed to follow a log-normal distribution [20]. Estimates for the mean differences in $(\log ) \mathrm{AUC}_{24}$ and $C_{\max }$ between milk and water were obtained for both study arms separately (with or without esomeprazole) using a linear mixed-effect model with treatment (water or milk), sequence and period as fixed effects, and subject-within-sequence as a random effect [21]. Variance components were estimated based on restricted maximum likelihood (REML) methods, and the Kenward-Roger method of computing the denominator degrees of freedom was used. The mean differences and their $95 \%$ confidence intervals (CIs) were exponentiated to provide point estimates of the ratio of geometric means and 95\% CIs for these ratios, which can be interpreted as relative differences in percentages. $T_{\max }$ was analyzed using the nonparametric Wilcoxon signed-rank test. Analyses to study the effect of esomeprazole were performed in a similar way, although they also included the effect of water versus milk as a fixed effect and only included patients who participated in both study arms. Toxicity was described as the incidence of toxicity per period. This was taken into account in case of 
an increase in CTCAE grade per cycle. Since the design of this study was not appropriate to detect a significant difference in toxicity, these results had a descriptive character. All statistical analyses were performed using Stata (StataCorp. 2017. Stata: Release 15.1. Statistical Software. College Station, TX, USA: StataCorp LP).

Table 1 Patient characteristics

\begin{tabular}{ll}
\hline Characteristic & Total included $[n=20]$ \\
\hline Sex & \\
Male & $7(35)$ \\
Female & $13(65)$ \\
Age, years [median (IQR)] & $67.5[55-73.5]$ \\
Performance status & \\
ECOG 0 & $10(50)$ \\
ECOG 1 & $10(50)$ \\
Race & \\
Caucasian & $16(80)$ \\
Asian & $3(15)$ \\
African & $1(5)$ \\
Current smoker & $0(0)$ \\
Erlotinib dose, mg & $17(85)$ \\
150 & $2(10)$ \\
100 & $1(5)$ \\
50 &
\end{tabular}

Data are expressed as $n(\%)$ unless otherwise specified

ECOG Eastern Cooperative Oncology Group, IQR interquartile range

\section{Results}

\subsection{Patients}

A total of 21 unique patients were included between February 2017 and November 2019. The patient demographics are presented in Table 1. For personal reasons, one patient withdrew informed consent after completion of the first period. Nine patients were included in both the non-PPI and PPI arms; hence, 29 pairs of study periods were completed-14 in the non-PPI arm and 15 in the PPI arm (Fig. 1).

\subsection{Pharmacokinetic Effects of Milk}

The pharmacokinetics of erlotinib when taken with milk or water are presented in Table 2. Erlotinib $\mathrm{AUC}_{24}$ decreased non-significantly by $3 \%(95 \% \mathrm{CI}-12$ to $8 \% ; p=0.567)$ when administered with milk, compared with water, in the nonPPI patients. In addition, in those patients who used esomeprazole, erlotinib exposure did not significantly differ as a result of intake with either water or milk $(0 \%$; $95 \%$ CI -15 to $17 \% ; p=0.953$ ). Figures $2 \mathrm{a}$ and $\mathrm{b}$ show the absence of an effect of milk in both study arms. $C_{\text {max }}$ did not differ in non-PPI or PPI users, with relative differences of a $6 \%$ and $1 \%$ increase, respectively ( $95 \% \mathrm{CI}-21$ to $11 \%, p=0.409$; and $95 \% \mathrm{CI}-12$ to $17 \%, p=0.831$, respectively). In both study arms, $T_{\max }$ increased non-significantly at $0.5 \mathrm{~h}$; in the non-PPI arm from 2.0 to $2.5 \mathrm{~h}(p=0.729)$ and in the PPI arm from 2.5 to $3.0 \mathrm{~h}(p=0.306)$. Interpatient variability, measured by the coefficient of variation $(\mathrm{CV})$, was lower with milk compared with water in both study periods and for both $\mathrm{AUC}_{24}$ and $C_{\text {max }}$. This lower variability in $\mathrm{AUC}_{24}$ with milk intake was most pronounced in the PPI arm (CV $38 \%$ vs. $61 \%$ ) [Table 2].

Table 2 Pharmacokinetic results per period

\begin{tabular}{|c|c|c|c|c|c|c|c|c|}
\hline $\begin{array}{l}\text { Pharmacokinetic } \\
\text { parameters }\end{array}$ & $\begin{array}{l}\text { No PPI with } \\
\text { water }[n=14]\end{array}$ & $\begin{array}{l}\text { No PPI with } \\
\text { milk }[n=14]\end{array}$ & $\begin{array}{l}\text { PPI with water } \\
{[n=15]}\end{array}$ & $\begin{array}{l}\text { PPI with milk } \\
{[n=15]}\end{array}$ & $\begin{array}{l}\text { RD, no-PPI with } \\
\text { milk vs. no-PPI } \\
\text { with water ( } 95 \% \\
\text { CI) }\end{array}$ & $p$ value & $\begin{array}{l}\text { RD, PPI with } \\
\text { milk vs. PPI } \\
\text { with water }(95 \% \\
\text { CI) }\end{array}$ & $p$ value \\
\hline \multicolumn{9}{|l|}{ Erlotinib } \\
\hline $\begin{array}{l}\mathrm{AUC}_{24}(\mathrm{CV} \%), \\
\text { geomean } \\
\mu \mathrm{g} * \mathrm{~h} / \mathrm{mL}\end{array}$ & $23.0(37)$ & $22.4(35)$ & $11.7(61)$ & $11.6(38)$ & $\begin{array}{l}-2.7 \%(-12 \text { to } \\
8 \%)\end{array}$ & 0.567 & $\begin{array}{l}-0.5 \%(-15 \text { to } \\
17 \%)\end{array}$ & 0.953 \\
\hline $\begin{array}{l}C_{\max }(\mathrm{CV} \%), \\
\text { geomean } \mu \mathrm{g} / \\
\mathrm{mL}\end{array}$ & $1.85(38)$ & $1.73(21)$ & $0.81(55)$ & $0.82(40)$ & $\begin{array}{l}-6.4 \%(-21 \text { to } \\
11 \%)\end{array}$ & 0.409 & $\begin{array}{l}1.5 \%(-12 \text { to } \\
17 \%)\end{array}$ & 0.831 \\
\hline $\begin{array}{l}T_{\max }(\mathrm{IQR}), \\
\text { median hours }\end{array}$ & $\begin{array}{l}2.00(1.52- \\
2.50)\end{array}$ & $\begin{array}{l}2.50(2.00- \\
3.00)\end{array}$ & $\begin{array}{l}2.52(2.05- \\
3.50)\end{array}$ & $\begin{array}{l}3.00(2.50- \\
3.52)\end{array}$ & NA & 0.729 & NA & 0.306 \\
\hline
\end{tabular}

$A U C_{24}$ area under the curve from time zero to $24 \mathrm{~h}, C I$ confidence interval, $R D$ relative difference, $C_{\max }$ maximum concentration, $C V$ coefficient of variation, $T_{\max }$ time until maximum concentration, IQR interquartile range, $N A$ not applicable, $P P I$ proton pump inhibitor 

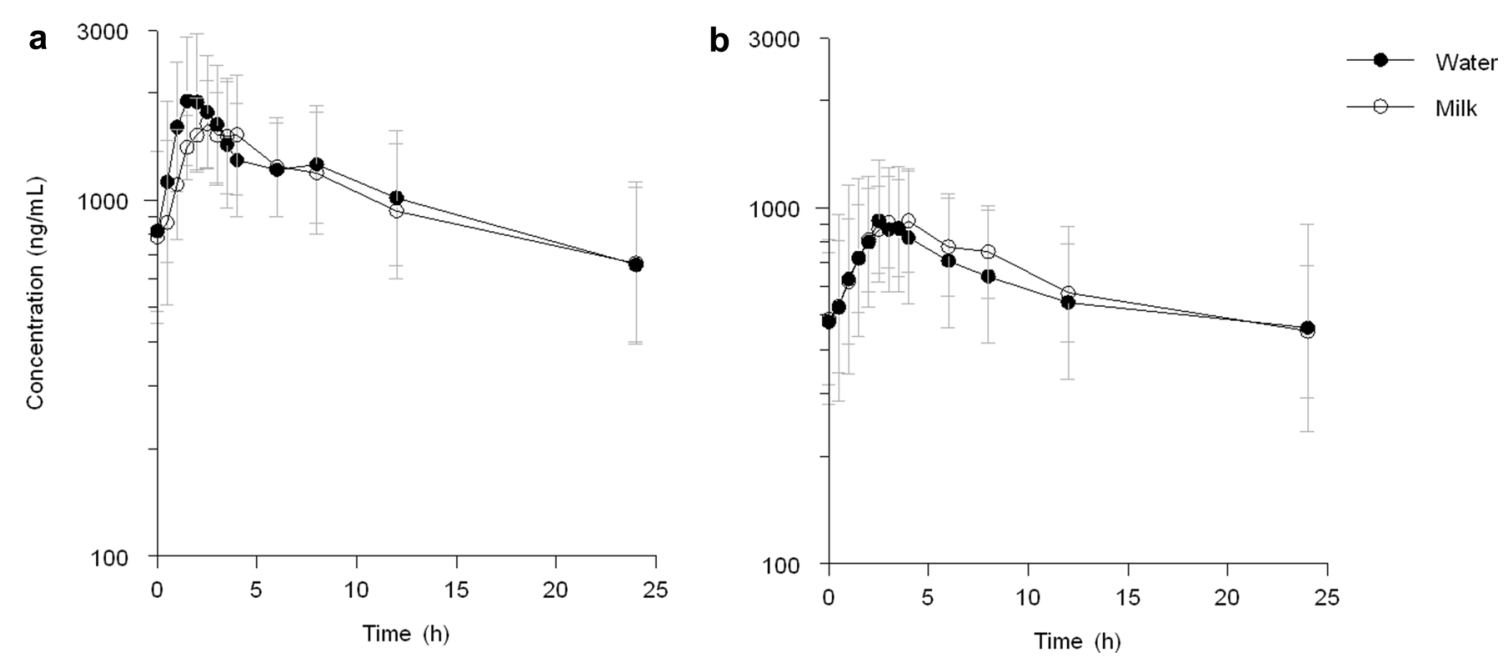

Fig. 2 Effect of cow's milk on erlotinib concentrations. Erlotinib taken with $250 \mathrm{~mL}$ of cow's milk or water, a without and b with concomitant esomeprazole administration

\subsection{Effects of Esomeprazole on Erlotinib Pharmacokinetics}

Based on data from the nine patients who participated in both study arms, esomeprazole decreased erlotinib $\mathrm{AUC}_{24}$ by an average of $47 \%(95 \% \mathrm{CI}-58$ to $-34 \% ; p<0.001)$ and $\mathrm{C}_{\max }$ by $56 \%(95 \% \mathrm{CI}-64$ to $-46 \% ; p<0.001)$ compared with the period in which esomeprazole was not used. These results are displayed in Fig. 3 and Table 3. $T_{\max }$ seemed longer for both the milk and water periods, especially in the PPI arm (Table 2). In the setting of administration with water, the interpatient variability in $\mathrm{AUC}_{24}$ increased from 37 to $61 \%$ due to esomeprazole co-treatment. When erlotinib was taken with milk, the interpatient variability in $\mathrm{AUC}_{24}$

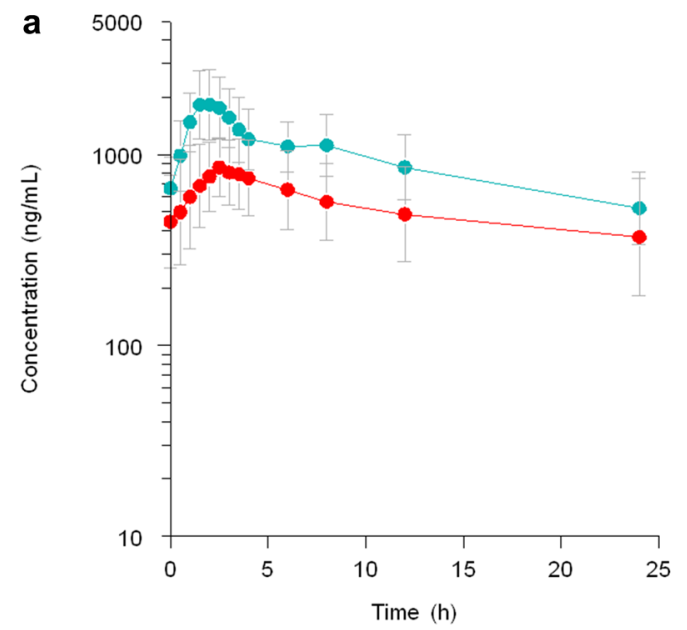

was not affected by esomeprazole co-treatment (CV 38\% vs. $35 \%$ ) [Table 2].

\subsection{Toxicity}

Table 4 presents all adverse events experienced. Overall, patient-reported adverse events during this study did not increase compared with baseline. Independent of study arm, no differences in toxicities were observed between study periods. Furthermore, patients reported almost equal adverse event grades in both the non-PPI and PPI arms (data not shown). Two grade 3 adverse events occurred—one period of nausea that fluctuated for several weeks, and one increase in skin rash during concomitant nadroparine treatment. Both patients used erlotinib for more than 3 months prior to this

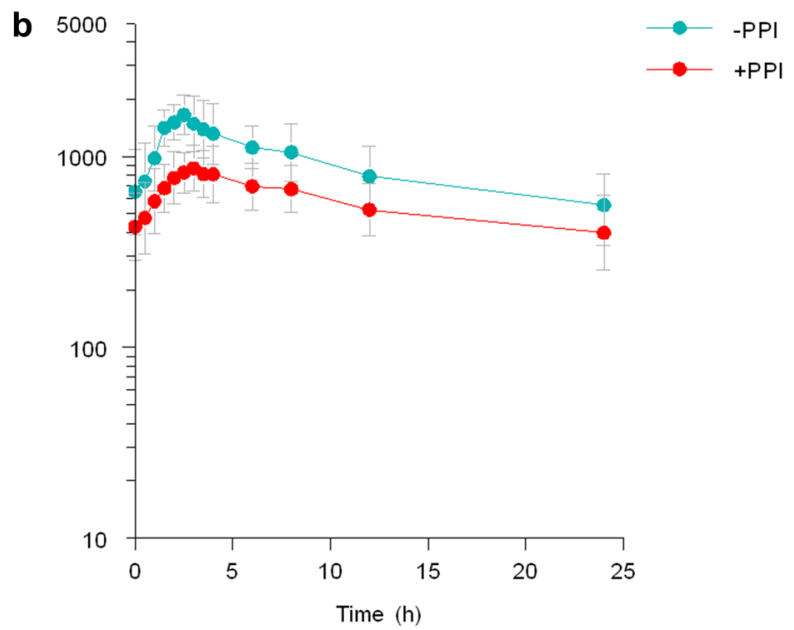

Fig. 3 Effect of esomeprazole on erlotinib concentrations. Erlotinib taken with a $250 \mathrm{~mL}$ water or b cow's milk. In the PPI arm, esomeprazole was administered $3 \mathrm{~h}$ prior to erlotinib intake. PPI proton pump inhibitor 
Table 3 Effect of esomeprazole on erlotinib pharmacokinetics

\begin{tabular}{lllll}
\hline Pharmacokinetic parameters & No PPI $[n=9]$ & PPI $[n=9]$ & RD, PPI vs. no PPI (95\% CI) & $p$ value \\
\hline Erlotinib & & & & \\
AUC $_{24}(\mathrm{CV} \%)$, geomean $\mu \mathrm{g} * \mathrm{~h} / \mathrm{mL}$ & $20.1(30)$ & $10.6(51)$ & $-47 \%(-58$ to $-34 \%)$ & $<0.001$ \\
$C_{\max }(\mathrm{CV} \%)$, geomean $\mu \mathrm{g} / \mathrm{mL}$ & $1.72(32)$ & $0.75(46)$ & $-56 \%(-64$ to $-46 \%)$ & $<0.001$ \\
\hline
\end{tabular}

Pharmacokinetic results for patients who participated in both study arms, corrected for coadministration with milk

$A U C_{24}$ area under the curve from time zero to $24 \mathrm{~h}, C I$ confidence interval. $R D$ relative difference, $C_{\max }$ maximum concentration, $C V$ coefficient of variation, $P P I$ proton pump inhibitor

Table 4 Patient-reported adverse events during the study period

\begin{tabular}{|c|c|c|c|c|c|c|}
\hline & Baseline $[n=30]$ & & Water $[n=29]$ & & Milk $[n=30$ & \\
\hline Adverse event & Grade $1-2$ & Grade 3 & Grade $1-2$ & Grade 3 & Grade $1-2$ & Grade 3 \\
\hline All events & $29(97)$ & - & $28(97)$ & - & $28(93)$ & $2(6)$ \\
\hline \multicolumn{7}{|c|}{ Reported in $\geq 10 \%$ of patients } \\
\hline Nausea & $1(3)$ & - & $3(10)$ & - & $3(10)$ & $1(3)$ \\
\hline Diarrhea & $6(20)$ & - & $3(10)$ & - & $3(10)$ & - \\
\hline Constipation & $5(17)$ & - & $1(3)$ & - & $3(10)$ & - \\
\hline Fatigue & $10(33)$ & - & $10(34)$ & - & $6(20)$ & - \\
\hline Pain & $5(17)$ & - & $7(24)$ & - & $9(30)$ & - \\
\hline Rash & $23(77)$ & - & $18(62)$ & - & $20(67)$ & $1(3)$ \\
\hline Alopecia & $12(40)$ & - & $11(38)$ & - & $11(37)$ & - \\
\hline Serious adverse event & - & $1(3 \%)^{\mathrm{a}}$ & - & - & - & - \\
\hline
\end{tabular}

Data are expressed as $n(\%)$

Water $=$ both periods wherein patients used water to take erlotinib, both without and with esomeprazole

Milk = both periods wherein patients used cow's milk to take erlotinib, both without and with esomeprazole

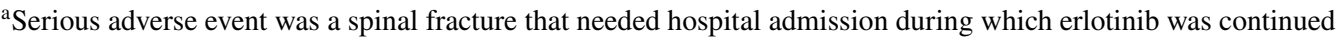

increase in toxicity. For the first patient, erlotinib was temporarily discontinued several weeks after study completion and restarted at a reduced dosage. For the second patient, erlotinib was temporarily discontinued and its dosage reduced. These dose reductions were effective in reducing toxicity in both cases. There was one serious adverse event (SAE) in this study, namely a CTCAE grade 3 malignant spinal fracture, which occurred after randomization and before the first study period. This SAE required hospital admission and was considered to be not related to study procedures, and erlotinib treatment was continued. No eminent study intervention-related toxicity occurred.

\section{Discussion}

This study reports the absence of a pharmacokinetic effect of cow's milk with $3.9 \%$ fat on exposure to erlotinib in NSCLC patients, independent of PPI use. Additionally, this study showed a decrease in erlotinib $\mathrm{AUC}_{24}$ of almost $50 \%$ and a decrease in $C_{\max }$ of more than $50 \%$ when erlotinib was administered $3 \mathrm{~h}$ after esomeprazole intake.
A possible explanation for the lack of effect of milk on erlotinib exposure is that the $3.9 \%$ fat content of cow's milk is not high enough to affect absorption. In absolute values, patients were administered $9.75 \mathrm{~g}(250 \mathrm{~mL} \times 3.9 \%)$ of fat from milk. This is relatively low in comparison with a highfat meal, which consists of 500-600 kilocalories of pure fat [3] (c.q. 56-67 g). The effect of a high-fat meal on erlotinib disposition ranges from a 33\% AUC increase when taken $2 \mathrm{~h}$ after erlotinib administration [22], to a $66 \%$ increase in AUC of erlotinib when food and drug are taken concomitantly [23]. In theory, the negative effect of esomeprazole of almost $50 \%$ decrease in $\mathrm{AUC}_{24}$ could be overcome by coadministration of a high-fat meal.

An additional reported effect of increasing the bioavailability of erlotinib with coadministration of a high-fat meal was a decrease in interpatient variability [3]. The benefits of less interpatient variability are a more predictable effectivity and toxicity on a large scale, since more patients will be administered within the therapeutic window. Our data show that milk also reduced interpatient variability, especially in the PPI arm (Table 2). Although, on average, bioavailability did not change, the lower interpatient variability would be 
an argument in favor of erlotinib administration with milk instead of water.

Another reason why erlotinib absorption was not affected by milk could be that the strong $\mathrm{pH}$ buffering capacity of milk [12] prevents the intragastric $\mathrm{pH}$ from decreasing. Hence, the beneficial effect of the milk's fat is counteracted by switching erlotinib to its less soluble, non-ionized form, which is not an optimal condition for transluminal transportation across gastrointestinal cells. Furthermore, there is no evidence of milk interacting with drug transporters or hepatic cytochrome $\mathrm{P} 450$ isoenzymes.

Average milk consists of 3-4\% fat [13]. Since we used cow's milk with the highest fat content (3.9\%) commercially available, it is unlikely that lighter variants of cow's milk would have a higher effect on the bioavailability of erlotinib. Nevertheless, cow's milk may be of interest for increasing systemic exposure of TKIs with vaster food effects, i.e. lapatinib (up to $325 \%$ and $200 \%$ AUC increase with a high- and low-fat meal, respectively) [3]. In line with milk, yoghurt (0.4\% fat [24]) is not expected to interact with erlotinib absorption and could also be considered safe. Coadministration with yoghurt was previously studied and was considered safe for the TKI nilotinib [3, 25].

Moreover, for the first time, we conducted an intrapatient comparison on the effects of esomeprazole on the $\mathrm{AUC}_{24}$ and $C_{\max }$ of erlotinib, which is in line with previous research with erlotinib and omeprazole [9]. We hence warn patients and prescribers of this possible harmful interaction, which could lead to therapy ineffectiveness. Potential solutions for patients who are dependent on PPI use may be a delayed PPI intake until erlotinib is fully absorbed or by taking erlotinib concomitantly with cola [10]. Albeit practical, the most feasible solution is a critical reconsideration of the need to prescribe a PPI and discontinuation of the PPI where possible.

Another way to increase the aqueous solubility, and therewith bioavailability, of erlotinib could be to improve its formulation [26]. A phospholipid formulation showed an improved pharmacokinetic profile in rats [27]. Before this new formulation could be considered to be implemented in clinical practice, further research should first be conducted to determine its possible benefits and deficits.

Furthermore, the absence of a milk effect on erlotinib exposure is probably also the reason why this study found no differences in patient-reported toxicity. This is not surprising as, for erlotinib, the plasma concentration is correlated with the occurrence of the most prevalent adverse effects of skin rash and diarrhea [28]. Erlotinib intake with milk is just as safe as intake with water, and could thus be advised to patients as an alternative for administration with water, for example to mitigate mild gastrointestinal reflux complaints or as the patient's preference.

Interestingly, although esomeprazole reduced erlotinib exposure by half, patients did not report less toxicity; however, the 3-day period during which patients had to take esomeprazole was most likely too short to have a noticeable effect on toxicity. When esomeprazole is taken for a longer period of time, the chronic decrease in erlotinib exposure could have a more distinctive effect of less toxicity.

\section{Conclusions}

Whole cow's milk with $3.9 \%$ fat has no clinically relevant effects on the exposure of erlotinib in NSCLC patients, independent of PPI use. The combination with milk instead of water is safe and well tolerated, and may be a good alternative for some patients. Meanwhile, the use of esomeprazole $3 \mathrm{~h}$ prior to erlotinib intake strongly decreased both erlotinib $\mathrm{AUC}_{24}$ and $C_{\max }$, and should be avoided if possible.

Acknowledgements The authors would like to acknowledge Peter de Bruijn for analyzing all study samples and for creation of the AUC curves for the figures.

Author contributions GDMV, KGAMH, RP, TvG, SLWK, RWFvL, JGJVA and RHJM designed the study. GDMV, KGAMH, KL and RHJM performed the research (c.q. screening and pharmacokinetic blood sampling). RP, CvdL, SDB, HBR, HNAB, CMJS, MSP, ACD and JGJVA selected patients for screening. GDMV, KGAMH and EOdH analyzed and interpreted the data. GDMV, KGAMH and RHJM wrote the manuscript. All other authors critically reviewed the manuscript and gave final approval for publication.

Funding This study was financially supported by an unrestricted grant from Roche.

Data Availability The datasets generated during and/or analyzed during the current study are available from the corresponding author upon reasonable request.

\section{Compliance with ethical standards}

Conflicts of Interest Christi M.J. Steendam reports grants from Roche, outside the submitted work; Anne-Marie C. Dingemans attended advisory boards and/or provided lectures for Roche, outside the submitted work (paid to institution); Teun van Gelder has received consulting fees from Roche Diagnostics, outside the submitted work; and Ron H.J. Mathijssen reports grants from Roche, outside the submitted work (paid to institution). G.D. Marijn Veerman, Koen G.A.M. Hussaarts, Robert Peric, Esther Oomen-de Hoop, Kersten D. Landa, Cor H. van der Leest, Suzanna D. Broerse, Hugo B. Rutten, Huub N.A. Belderbos, Marthe S. Paats, Stijn L.W. Koolen, Roelof W.F. van Leeuwen and Joachim G.J.V. Aerts declare no competing interests. (Part of) this work was presented at ESMO 2019 as a poster presentation (\#1540P).

Ethics Approval This study was approved by the local Ethics Committee (Erasmus University Medical Center Rotterdam; MEC 16-590) and was registered in the Dutch Trial Registry (number NL5984; NTR6148).

Consent to Participate All participating patients were asked to sign a written informed consent form. 
Open Access This article is licensed under a Creative Commons Attribution-NonCommercial 4.0 International License, which permits any non-commercial use, sharing, adaptation, distribution and reproduction in any medium or format, as long as you give appropriate credit to the original author(s) and the source, provide a link to the Creative Commons licence, and indicate if changes were made. The images or other third party material in this article are included in the article's Creative Commons licence, unless indicated otherwise in a credit line to the material. If material is not included in the article's Creative Commons licence and your intended use is not permitted by statutory regulation or exceeds the permitted use, you will need to obtain permission directly from the copyright holder. To view a copy of this licence, visit $\mathrm{http} / / /$ creativecommons.org/licenses/by-nc/4.0/.

\section{References}

1. US FDA. Erlotinib product review and label. Available at: https ://www.accessdata.fda.gov/scripts/cder/daf/

2. Planchard D, et al. Metastatic non-small cell lung cancer: ESMO Clinical Practice Guidelines for diagnosis, treatment and followup. Ann Oncol. 2018. 29 Suppl 4:iv192-iv237. Available at: https ://www.esmo.org/Guidelines/Lung-and-Chest-Tumours/Metas tatic-Non-Small-Cell-Lung-Cancer (last updated 18 Sep 2019).

3. Veerman GDM, et al. Clinical implications of food-drug interactions with small-molecule kinase inhibitors. Lancet Oncol. 2020;21(5): $265-79$.

4. Hussaarts $\mathrm{K}$, et al. Clinically relevant drug interactions with multikinase inhibitors: a review. Ther Adv Med Oncol. 2019;11:1758835918818347.

5. Mathijssen RHJ, Sparreboom A, Verweij J. Determining the optimal dose in the development of anticancer agents. Nat Rev Clin Oncol. 2014;11(5):272-81.

6. O'Malley M, et al. Effects of cigarette smoking on metabolism and effectiveness of systemic therapy for lung cancer. J Thorac Oncol. 2014;9(7):917-26.

7. Honeywell RJ, et al. Transport of six tyrosine kinase inhibitors: active or passive? ADMET DMPK. 2016;4:23-34.

8. van Leeuwen RW, et al. Drug-drug interactions with tyrosine-kinase inhibitors: a clinical perspective. Lancet Oncol. 2014;15(8):e315-26.

9. Kletzl H, et al. Effect of gastric $\mathrm{pH}$ on erlotinib pharmacokinetics in healthy individuals: omeprazole and ranitidine. Anticancer Drugs. 2015;26(5):565-72.

10. van Leeuwen RW, et al. Influence of the acidic beverage cola on the absorption of erlotinib in patients with non-small-cell lung cancer. J Clin Oncol. 2016;34(12):1309-14.

11. Singh N, Prasad KT. A cola to improve my EGFR TKI absorption in metastatic non-small-cell lung cancer? No, thank you, I prefer lemonade or orange juice instead. J Clin Oncol. 2016;34(33):4053-4.
12. Charkoftaki G, Kytariolos J, Macheras P. Novel milk-based oral formulations: proof of concept. Int J Pharm. 2010;390(2):150-9.

13. Food and Agriculture Organization of the United Nations. Milk and dairy products in human nutrition, 2013. E-ISBN 978-92-5107864-8. Available at: https://www.fao.org/3/i3396e/i3396e.pdf.

14. Oken MM, et al. Toxicity and response criteria of the Eastern cooperative oncology group. Am J Clin Oncol. 1982;5(6):649-55.

15. The Netherlands Trial Register. Available at: https://www.trial register.nl/.

16. van Leeuwen RWF, et al. Tyrosine kinase inhibitors and proton pump inhibitors: an evaluation of treatment options. Clin Pharmacokinet. 2017;56(7):683-8.

17. Braal CL, et al. Quantification of the tyrosine kinase inhibitor erlotinib in human scalp hair by liquid chromatography-tandem mass spectrometry: pitfalls for clinical application. J Pharm Biomed Anal. 2019;172:175-82.

18. US Department of Health and Human Services. Common Terminology Criteria for Adverse Events (CTCAE) Version 4.03. 14 June 2010. Bethesda, MD: US National Institute of Health.

19. Schoenfeld DA. Statistical Considerations for a Cross-Over Study. Available at https://hedwig.mgh.harvard.edu/sample_size/js/js_ crossover_quant.html.

20. European Medicines Agency. Guideline on the Investigation of Bioequivalence. 20 January 2010. Available at: https://www.ema. europa.eu/en/documents/scientific-guideline/guideline-investigat ion-bioequivalence-rev1_en.pdf.

21. Jones B. Design and analysis of cross-over trials. 2nd ed. United States: Chapman \& Hall/CRC; 2003.

22. Katsuya $Y$, et al. Comparison of the pharmacokinetics of erlotinib administered in complete fasting and $2 \mathrm{~h}$ after a meal in patients with lung cancer. Cancer Chemother Pharmacol. 2015;76(1):125-32.

23. Ling $\mathbf{J}$, et al. Effect of food on the pharmacokinetics of erlotinib, an orally active epidermal growth factor receptor tyrosine-kinase inhibitor, in healthy individuals. Anticancer Drugs. 2008;19(2):209-16.

24. US Department of Agriculture. Yogurt, Greek, plain, nonfat (includes foods for USDA's Food Distribution Program). Available at: https://fdc.nal.usda.gov/fdc-app.html\#/food-details/33013 7/nutrients.

25. Yin OQ, et al. Effects of yogurt and applesauce on the oral bioavailability of nilotinib in healthy volunteers. J Clin Pharmacol. 2011;51(11):1580-6.

26. Sawicki E, et al. Inventory of oral anticancer agents: pharmaceutical formulation aspects with focus on the solid dispersion technique. Cancer Treat Rev. 2016;50:247-63.

27. Dora CP, et al. Improved oral bioavailability and therapeutic efficacy of erlotinib through molecular complexation with phospholipid. Int J Pharm. 2017;534(1-2):1-13.

28. Fiala $\mathrm{O}$, et al. Serum concentration of erlotinib and its correlation with outcome and toxicity in patients with advanced-stage NSCLC. Anticancer Res. 2017;37(11):6469-76.

\title{
Affiliations
}

\author{
G. D. Marijn Veerman ${ }^{1} \cdot$ Koen G. A. M. Hussaarts ${ }^{1} \cdot$ Robert Peric $^{2} \cdot$ Esther Oomen-de Hoop $^{1} \cdot$ Kersten D. Landa $^{1}$. \\ Cor H. van der Leest ${ }^{3}$. Suzanna D. Broerse $e^{4}$ Hugo B. Rutten ${ }^{5}$. Huub N. A. Belderbos ${ }^{3}$. Christi M. J. Steendam ${ }^{2,3}$. \\ Marthe S. Paats ${ }^{2}$. Stijn L. W. Koolen ${ }^{1,6} \cdot$ Anne-Marie C. Dingemans ${ }^{2} \cdot$ Teun van Gelder ${ }^{6} \cdot$ Roelof W. F. van Leeuwen $^{1,6}$. \\ Joachim G. J. V. Aerts ${ }^{2} \cdot$ Ron H. J. Mathijssen ${ }^{1}$
}


1 Department of Medical Oncology, Erasmus MC Cancer Institute, Erasmus University Medical Center, Dr. Molewaterplein 40, 3015 GD Rotterdam, The Netherlands

2 Department of Pulmonology, Erasmus MC, Rotterdam, The Netherlands

3 Department of Pulmonology, Amphia Hospital, Breda, The Netherlands
4 Department of Pulmonology, Franciscus Gasthuis and Vlietland Hospital, Rotterdam, The Netherlands

5 Department of Pulmonology, Bravis Hospital, Roosendaal and Bergen op Zoom, The Netherlands

6 Department of Hospital Pharmacy, Erasmus MC, Rotterdam, The Netherlands 